policy on young adults to educate them on harms of alcohol and minimise consequences of harmful use of alcohol for a healthy population in the long run.

\section{THE IMPACT OF THE DIGITALIZATION TO THE MEDICAL SUPPLY CHAIN}

Teijo Peltoniemi, Reima Suomi. University of Turku, Finland

10.1136/injuryprev-2016-042156.555

Background Digitalization equalises information asymmetries, which increases economic efficiency and transforms many lines of business. We argue that digitalization can do the same within the medical supply chain.

Methods We utilise conceptual theory building. This is supported by a literature review and an interview conducted with a key actor within the Finnish pharmaceutical arena.

Results 1) Electronic prescription has the potential to increase the information between physician and patient. Some positive results have been obtained relating to medication safety. 2) The generic substitution system has been implemented to decrease the information asymmetry relating to medicine prices. This has had an effect on the annual medicine expenditures in Finland. 3) These developments have however no influence to the lack of patient adherence. It is a form of information asymmetry with a grave impact to the healthcare system. Adherence could be alleviated with digital solutions. These include solutions supporting patient engagement as well as controlled delivery of medicines at home. 4) Outcomes-based medicine tries to decrease the uncertainty in the field of medical care. This can be alleviated with data gathered from the patient with innovations such as wearables. This can have impact also to the moral hazard threat in the health insurance field. 5) Overall, there is more information available on medical treatments, which should be utilised to increase the patient engagement. 6) Medicine spoilage and shortage are severe information asymmetry related problems. Digital systems could support the prediction of the demand and real time tracking of the consumption of medicines.

Conclusions Digitalization could improve the medical supply chain by decreasing the information asymmetries between different actors. Whereas there is some promising development, considerable potential remains still unused.

\section{PREVALENCE AND FACTORS ASSOCIATED WITH WORK- RELATED INJURY AMONG 40/60 CONDOMINIUM CONSTRUCTION WORKERS IN ADDIS ABABA, ETHIOPIA}

Dagnachew Israel, Sebsibie Tadesse, Mekuriaw Alemayehu. University of Gondar, Ethiopia

10.1136/injuryprev-2016-042156.556

Background Injuries pose a major public health and development problems in construction work places. However there is limited studies and given less attention by the government and companies to protect the construction workers from injury. This study aims to estimate prevalence and factors associated with injury among 40/60 condominium construction workers in Addis Ababa, Ethiopia.

Methods A cross-sectional quantitative data method was conducted to estimate the prevalence and factors associated with injury among 40/60 condominium construction workers. For the survey, 504 workers from city government of Addis Ababa 40/60 condominium sites were selected using multistage sampling technique. Data were collected using pre-tested and structured questionnaire. The data were entered by using EPI Info version 7 and analysed using SPSS version 20, and results were presented in tables and figures. Odds ratio with $95 \%$ confidence interval was used as a measure of effect.

Result The prevalence is $38.3 \%$, of the respondents have got injuries. Marital status (married of [AOR: $=0.054(0.005-0.616)]$, single AOR: $=0.058(0.005-0.661)$, Divorcee [AOR: $=0.072$ (0.005-0.0989)] and coupled [AOR: $=0.031(0.002-0.403)]$, service [AOR: $=0.404(0.231-0.711)]$, chewing chat [AOR: $=$ $2.570(1.589-4.158)]$ and not using personal protective device [AOR: $=0.355(0.192-0.654)]$ were significantly associated with injury among 40/60 condominium construction workers.

Conclusion In this study the prevalence of injury among 40/60 construction workers was $38.3 \%$ CI 95\% $(33.9,42.5)$ with significant associated factors of marital status, chat chewing, service year and not using personal protective device. Therefore counter measures such as creating awareness of risk factors, providing and using personal protective devices could be effective to decrease prevalence of injuries among construction workers.

\section{PRESCRIBED MEDICATION AND ADVERSE DRUG EVENTS BY UNINTENTIONAL POISONING AMONG OLDER ADULTS: A SWEDISH POPULATION-BASED MATCHED CASE-CONTROL STUDY}

${ }^{1,2}$ Christian Rausch, 'Lucie Laflamme, 'Jette Möller. 'Karolinska Institutet Sweden; ${ }^{2}$ University Medical Centre Groningen, The Netherlands

\subsection{6/injuryprev-2016-042156.557}

Background Specific types of medications, but also drug-drug interactions are known to lead to adverse drug events (ADE) among older people. However, only small numbers of controlled epidemiological studies investigated ADE with particular focus on the prescription of inappropriate medications (inappropriate drug use - IDU) or the number of medications prescribed. This based study investigates the association between the number of prescribed medications and ADEs, while considering different indicators of inappropriate drug use (IDU).

Methods In this study we utilised a matched case-control design among Swedish residents 50 years and older. The National Inpatient Register and the Causes of Death Register were used to extract cases with and ADE by unintentional poisoning that resulted in a hospitalisation or death within January 2006 to December 2009 ( $n=5,336)$. Each case was matched with four controls, based on age, sex and residential area. The controls were randomly selected among the individuals without an ADE ( $n=21,344)$. Any medication prescribed during the four-month period prior to the ADE were selected by using the Swedish Prescribed Drug Register and were then categorised based on the total number of medications prescribed and on correspondence with an IDU indicator (known single-drug and drug combinations). Effects were estimated with odds ratios (OR) and 95\% confidence intervals (CI:) by applying conditional logistic regression.

Results Each of the indicators for IDU was associated with a high risk of ADE among older people. We found a lower, yet still positive and graded association between the total number of medications prescribed and the risk for ADE, starting by two medications to ten or more. The risk of ADE remained increased when the total number of medications prescribed was at three to 
ten or more medications, even after exclusion of IDU indicators from the total number of medications prescribed.

Conclusions The total number of medications and IDU indicators are associated with ADE among older people. Although IDU indicators were positively associated with ADE, they lacked to fully explain the graded association with increasing numbers of medications prescribed. Physicians, but also patients need to be aware of this increased likelihood of serious ADE by low and increasing numbers of medications prescribed.

\section{EDUCATIONAL INTERVENTIONS DESIGNED TO IMPROVE MEDICATION ADMINISTRATION SAFETY}

${ }^{1}$ Marja Härkänen, ${ }^{1}$ Ari Voutilainen, ${ }^{2}$ Elina Turunen, ${ }^{1,2}$ Katri Vehviläinen-Julkunen. ${ }^{1}$ University of Eastern Finland, Finland; ${ }^{2}$ Kuopio University Hospital, Finland

10.1136/injuryprev-2016-042156.558

Background Increasing the medication administration safety is vital to improving patient safety. The aim of this study is to analyse educational interventions designed to increase the medication administration skills and safety of registered nurses.

Methods A systematic review of the literature using six databases was conducted to identify intervention studies published between January 2000 and April 2015. The quality of studies was assessed using the Effective Public Health Practice Project (EPHPP) Quality Assessment Tool. The efficacy of the interventions was determined by calculating the effect sizes and conducting a metaanalysis.

Results Fourteen intervention studies were included in this systematic review. Quality was strong only in one study and moderate in another four. The interventions differed significantly from one study to the next, and the original investigators reported significant improvement in the skills and medication safety owing to the interventions. The meta-analyses, however, revealed that the effect of the intervention was strong in only four of the studies. Those interventions were the 60-minute educational intervention (PowerPoint presentation), a blended learning programme inclusive of e-learning, wall poster and informative pamphlets, and a combination of three different interventions (classroom or selfstudy methods). In two out of four cases, the quality of the study was weak and moderate in two cases, and only one of the effective interventions was a randomised controlled trial.

Conclusions Based on the original analysis, all the interventions reviewed had a positive impact on medication administration safety. Nonetheless, these positive outcomes do not necessarily indicate effective interventions. The most effective interventions in this review were methodologically dissimilar. Studies should be assessed for their quality and a meta-analysis performed when searching for the best, effective and highest quality educational interventions.

\section{EXAMINING THE RELATIONSHIP BETWEEN EDUCATION AND ALCOHOL-ATTRIBUTABLE NON-FATAL INJURY RISK IN BRAZIL}

${ }^{1}$ Deena El-Gabri, ${ }^{1}$ Nicole Toomey, ${ }^{1,2}$ João Ricardo Vissoci, ${ }^{1,3}$ Catherine Staton. ' Duke Global Health Institute; ${ }^{2}$ Faculdade Ingá; ${ }^{3}$ Duke University Medical Centre

10.1136/injuryprev-2016-042156.559

Background In Brazil, alcohol use is a leading risk factor for injury contributing to over $8 \%$ of DALYs lost. While alcohol consumption increases with income, there is an inverse risk of alcohol-attributable mortality with socioeconomic status (SES). Education is an important measure of SES; yet its effect on risk for non-fatal injury is unclear. This project investigates education level most at risk for alcohol positive injury in Maringá, Brazil.

Methods Self-reported alcohol usage two hours prior to injury was collected during a survey on treatment-seeking behaviour following injury in Maringá, Brazil between May and September 2015. Households provided demographics, and one randomly selected household member supplied injury history. Alcohol usage prior to injury and demographics were analysed using frequencies and logistic regression. Risk ratios were calculated using primary school incomplete, the lowest risk group, as reference.

Results Of 2678 people surveyed, 797 reported injury. Only 56 (7.02\%) reported alcohol use of which $85.7 \%$ were male. Of all alcohol-attributable injured patients, 3 (5.4\%) did not complete primary school, $8(14.3 \%)$ completed primary school, 12 (21.4\%) completed secondary school, and 15 (26.8\%) completed professional school. Those who completed primary education and professional education were at similar risk for alcohol-attributable injuries. Completing primary school $(\mathrm{RR}=4.14$, $\mathrm{p}=0.032)$ and professional school $(\mathrm{RR}=3.43, \mathrm{p}=0.047)$ had the highest risk of alcohol-induced non-fatal injury.

Conclusions Alcohol use is a major risk factor for injury and is influenced by education. While our sample size is small and only includes non-fatal injuries, it mirrors the general pattern of alcohol usage seen in Brazil. We found low and high levels of education were at highest risk for alcohol-related injury. Our data suggests an indirect dependence of education on alcohol-induced injury however this relationship requires more in depth research.

\section{ALCOHOL LEVELS AND INJURY RELATED MORTALITY}

Ms Aine O'Brien, Michal Molcho. School of Health Sciences, NUI Galway, Ireland

\subsection{6/injuryprev-2016-042156.560}

Background Limited data on injury mortality were traditionally collected using death certificates. Coroners' inquest reports offer a richer source of injury mortality data; collating information from multiple sources (including police reports, witness statements, coronial autopsy and forensic pathologist post-mortem and toxicology examinations). These reports provide information on the manner and mechanism of injury, the presence of alcohol and drugs, a detailed description of injuries sustained and a narrative of the circumstances resulting in death.

Methods This study reviewed 722 coroners' inquest investigation reports of injury related death between 2006 and 2010 in the West of Ireland, including coronial and forensic pathologist postmortem and toxicology examination results. Alcohol levels were measured in blood and urine and included in toxicology results. This paper focuses on the presence of alcohol in various injury related fatalities.

Results Alcohol was detected in $54.2 \%$ of cases $(78 \%$ male and $22 \%$ female), most commonly among 0-44 year-olds (49\%). Where alcohol was present, it was most frequently at a level greater than $200 \mathrm{mg} / 100 \mathrm{ml}$. Alcohol was present in $41 \%$ of unintentional fatalities, $19 \%$ of intentional fatalities, and $40 \%$ of undetermined intent fatalities. Alcohol was detected in $40 \%$ of asphyxiated fatalities, consisting of $49.7 \%$ hanging and $41.4 \%$ of drowning fatalities. Blood alcohol levels $>200 \mathrm{mg} / 100 \mathrm{ml}$ were found in $39 \%$ of the drowning fatalities, while levels $<20 \mathrm{mg} / 100$ $\mathrm{ml}$ were detected in $30 \%$ of the hanging fatalities. 